\title{
VICO A MI MANERA
}

\section{Miguel A. Pastor Pérez (Universidad de Sevilla)}

RESUMEN: Un Vico multiforme, pluriversal, polifacético, es el Vico que el Autor plantea a partir de las propias lecturas de la obra del napolitano, de las interpretaciones de los muy distintos autores que han afrontado tanto al filósofo napolitano, como su obra, su vida, su figura. Un Vico dado también a través de la traducción, de la re-construcción de su propio pensar, tamizado por distintas tradiciones culturales, diferentes orientaciones hermenéuticas e historiográficas, diversas aproximaciones personales.

Palabras Clave: Vico, $350^{\circ}$ Aniversario, obra, figura, pensamiento, traducción, tradición, cultura, Cuadernos sobre Vico, M.A. Pastor Pérez.

\section{Vico my way}

ABSTRACT: A multiform, pluriversal, multifaceted Vico, is the one that the Author raises from his own readings of the work of the Neapolitan and from the interpretations of the very different authors who have confronted both the Neapolitan philosopher, his work, his life and his figure. A Vico that is also given through translation and through the re-construction of his own thinking, sifted by different cultural traditions, different hermeneutical and historiographical orientations, different personal approaches.

KeYwords: Vico, $350^{\text {th }}$ Anniversary, work, figure, thought, translation, tradition, culture, Cuadernos sobre Vico, M.A. Pastor Pérez.

\section{Vico a modo mio}

RiASSUNTO: Un Vico multiforme, pluriversale, poliedrico, è il Vico che l'Autore espone a partire dalle proprie letture dell'opera del filosofo napoletano e dalle interpretazioni dei diversi autori che hanno affrontato lo studio del pensatore napoletano, la sua opera, la sua vita e la sua figura. Un Vico che emerge anche dalla traduzione, dalla ricostruzione del proprio pensiero, vagliato da tradizioni culturali differenti, da orientamenti ermeneutici e storiografici distinti e da diversi approcci personali.

Parole ChiAVE: Vico, $350^{\circ}$ Anniversario, opera, figura, pensiero, traduzione, tradizione, cultura, Cuadernos sobre Vico, M.A. Pastor Pérez.

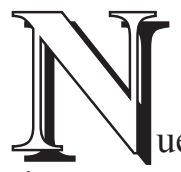

uestra relación intelectual con Giambattista Vico es larga, intensa, productiva, sugerente, polémica a veces, aunque casi siempre mediata.

Este artículo responde a una invitación expresa por parte de la Dirección de la Revista para este volumen especial por el $350^{\circ}$ Aniversario del nacimiento de G. Vico, habiendo superado los criterios de valoración y del proceso de aceptación. 
Los inicios de esta relación se remontan a bastantes años atrás, cuando el autor de estas reflexiones era apenas un adolescente pre-univesitario, devorador omnívoro de cualquier material impreso, y en cuyas manos cae, a través de una Enciclopedia familiar de inicios de los años ' 70 que estaba presente en muchas casas de la naciente clase media española de la época, una primera referencia a la Ciencia nueva de Vico, que en mi memoria, y sin recordar muy bien por qué, tal vez algún criterio emocional más que posicional u organizativo, siempre estuvo asociada a La Araucana de Alonso de Ercilla. La Enciclopedia en cuestión era de la editorial Montaner y Simón, que proveerá luego de otro de los grandes hitos, en opinión del Autor, de la difusión filosófica como será la Historia de la Filosofía de N. Abbagnano, que también ejercerá una notable influencia.

Comenzados ya los estudios universitarios, la presencia de Vico se patentiza en un curso de "Filosofía de la Historia" (1979) de la mano del profesor José Luis López López y posteriormente a través de la realización de mi "tesina" de licenciatura (1984) sobre el abate Esteban de Arteaga (1747-1799) - exiliado jesuita incompleto, escritor crítico, esteta y musicólogo por convicción- y su relación intelectual y conceptual con algunas de las propuestas estéticas de G.B. Vico (1688-1744), coetáneos ambos de un siglo de transición, que permitirá a Arteaga resituarse en una posición pre-romántica que lo lleva a concebir el «teatro musicale italiano», o sea la Ópera, como un Arte Total que hace del arte escénico musical la forma máxima de expresión de la sensibilidad humana, y en el cual están implicadas la mayoría del resto de las Artes.

El napolitano nos ayudará a comprehender la evolución de la Ópera hasta llegar a constituirse como Arte total, expresión última y máxima de todo un proceso de desarrollo e integración mediante las distintas artes, que expresan históricamente el acceso del sujeto, de la humanidad al ámbito del gusto, de la sensibilidad, de la estética, en adecuación al progreso cultural y social de las naciones, de las gentes siguiendo los principios de la historia ideal eterna mediante el mecanismo de los corsi-ricorsi.

Será en 1991, con la aparición de la revista Cuadernos sobre Vico -auspiciada inicialmente, entre otros, por el "Centro de Investigaciones sobre Vico" y por la propia Universidad de Sevilla, y dirigida la publicación por el prof. José M. Sevilla-, cuando esta relación mediada entre Vico y el autor de estas notas se hará más intensa, pero también más extensa, a partir del hecho de ocupar el puesto de Director Adjunto de la recién nacida Revista.

La gestación de una revista científica especializada -seguro que algunos de los lectores lo saben por experiencia- exige un inmenso trabajo previo, tanto conceptual, relacional y académico, como logístico-material. Jugábamos además con el hándicap de ser una revista que pretendía, sin dejar de ser rigurosamente científica, centrarse -que no ceñirse- en un ámbito cultural, un tema científico y un autor 
determinado, y que quería además publicar siempre en español a partir de los originales provenientes de cualquier parte del mundo en cualquier idioma.

De aquí, en definitiva, que durante dos años y a través de los fundadores del "Centro de Investigaciones sobre Vico", y especialmente por el empeño y esfuerzo del prof. Sevilla, se fuese pergeñando un determinado "concepto" de medio de difusión, de revista científica, que reuniese las condiciones de calidad y rigor intelectual propios sobre el tema en cuestión y siguiendo la estela hermana de otras publicaciones especializadas, algunas de las cuales ya han desaparecido. Nos referimos aquí, obviamente, al Bollettino del Centro di studi vichiani, fundado por P. Piovani en 1971 y dirigido brillantemente por varios directores a lo largo de sus diversas épocas; y a los New Vico Studies auspiciados por G. Tagliacozzo en 1983 y tutelados y dirigidos más adelante por el prof. D.Ph. Verene de la Universidad de Emory. La publicación americana desapareció en 2010.

Así, en 1991, aparece en la ciudad de Sevilla y desde su Universidad el número 1 de Cuadernos sobre Vico, con la firme vocación y voluntad de integrarse a nivel científico en el océano de publicaciones, tanto especializadas como de divulgación, sobre temas humanistas y filosóficos y en las que sobrenadaban los pensamientos y la reflexión filosófica. Se alumbraba así un Vico pensado "en español", con un amplio espacio geográfico, conceptual y cultural de producción, pero también de incidencia, que venía a acompañar, completar y dinamizar los consolidados Vico en italiano y Vico anglo-americano vertidos en las publicaciones vehiculares correspondientes.

A partir de aquí, la intrahistoria intelectual, académica y personal entre el autor napolitano y quien esto suscribe está jalonada de "hitos". El primer hito lo constituye la publicación definitiva de ese primer número, con sus limitaciones inherentes, de la Revista. Número elaborado únicamente a partir de artículos de autores españoles, que en un primer momento consideraron oportuno confiar en el desarrollo y consolidación de la publicación. A través de las traducciones de otros autores, a través de la lectura preparatoria de las distintas propuestas y temas, de la composición formal de los números, se abría y dibujaba un Vico pluriversal y de múltiples caras, que cambiaba conforme era enfrentado por las distintas interpretaciones y formas diversas de comprender su mundo, su pensamiento en sus distintas facetas, desde la metafísica hasta la estética, pasando por la histórica, la lingüística, la moral, la poética, la jurídica y la política. Versiones múltiples que nos permitieron conocer un Vico diferente. Más allá de sus propios escritos, más allá de consideraciones tradicionales, más allá de interpretaciones vanguardistas, más allá de su aislamiento y más allá de la afirmación de su integración europea, Vico se nos revelaba en un constante proceso de renovación, bajo una forma variante de corsi y ricorsi, tan queridos por él, que permitía tener acceso a una casi infinita variedad de matices sobre su pensamiento, además de que lo actualizaban constantemente. 
Complemento hito de este número inicial, de marcado carácter hispánico, basado en la confianza en la posibilidad de desarrollar todos esos matices intuidos del pensamiento viquiano desde nuestra perspectiva, va a ser la aparición del número 2 de la Revista, de acentuada condición europea e internacional en esta segunda oportunidad a pesar de ser solo cuatro los autores foráneos ${ }^{1}$ y cuya presencia como manifestación de apoyo al proyecto resultará a la larga fundamental, y en el que a su vez, a las aportaciones de algunos autores fundamentales dentro de la tradición crítica respecto a Vico habría que añadir, en mi caso, el acercamiento, el acceso, la propuesta de conocer otros aspectos del pensamiento del autor napolitano a través de la preparación logística del número, a través de la búsqueda de traductores cualificados, de la lectura y preparación editorial de los distintos artículos y trabajos, tanto sobre Vico como sobre la época y la cultura hispano-italiana, y sus diversas posibles temáticas a través de la lectura original necesaria para la revisión de artículos y propuestas editoriales.

Recordamos, precisamente, el problema planteado a nivel de translación idiomática por el artículo del prof. G. Costa sobre las «sali nitri» de Vico (en Cuadernos sobre Vico, $\mathrm{n}^{\circ}$ 2) como elementos constitutivos de los "gigantes", término contrafáctico, a su vez, fundamental para entender globalmente el papel de la antropología en la constitución de los principios que rigen la naturaleza de las naciones como apela la obra principal de nuestro autor. O el papel de la icono-logía/iconografía en la obra de Vico tal y cómo ha sido seguida por varios autores. O la investigación derivada de la problemática que ha rodeado el carácter apócrifo y la forma de la presencia de Vico en la web a partir de un determinado retrato (ibíd., nº 23).

El elenco de especialistas, estudiosos y autores interesados en el pensamiento de Vico, y que han escrito en la presente Revista, es inmenso y su procedencia singularmente internacional, tanto desde el ámbito hispanoamericano como el anglosajón en América, tanto desde Europa del norte como del sur, tanto de Occidente como de Oriente (Israel o Japón). Además de las aportaciones inherentes a las propuestas conceptuales, a las interpretaciones históricas y sistemáticas, a las orientaciones sugeridas de lectura directa de los textos viquianos, el trabajo editorial que supone no solo trabajar con los autores sino también con el propio pensamiento y obra de Vico nos ha enseñado o mostrado "otros" Vico, mediados por su propia palabra en muchos casos, y otras veces sin llegar a estar de acuerdo conceptualmente, pero siempre leídos desde el intento de objetivar, mostrar y verificar las singulares propuestas del autor napolitano tanto en su propio tiempo como más allá del mismo, así como de su influencia en el nuestro, un tiempo nuestro que se actua-

1. Queremos dejar constancia de estos autores por lo que significarán como argumento de apoyo al proyecto y su alcance posterior, así como su experto conocimiento del pensamiento de Vico y sus ámbitos epistemológicos. Nos referimos a los profesores Gustavo Costa, Ernesto Grassi -fallecido en el mismo año 1991-, Emilio Hidalgo-Serna -de origen español aunque profesor de la Academia alemana- y Fulvio Tessitore. 
liza constantemente (ya hemos publicado el número 31 de Cuadernos sobre Vico celebrando en el pasado año 2017 el XXV aniversario de la Revista).

Otro hito fundamental personal -como Secretario que fui del evento- para entender el pensamiento de Vico como una totalidad llena de sentido, viene dado por la celebración en 1999, también en la ciudad de Sevilla, de uno de los Congresos más importantes sobre el pensamiento de Vico habidos hasta el momento en el ámbito internacional y, desde luego, en el mundo hispánico. Nos referimos al Congreso Internacional: Pensar para el nuevo siglo. G.B. Vico y la cultura europea, llevado a cabo en los días 4-9 de Octubre, y en el que se desarrollaron 52 ponencias magistrales por parte de los más reconocidos especialistas, estudiosos, investigadores y expertos de todo el mundo (Italia, España, Alemania, USA, Argentina, Canadá, Brasil, Israel, Francia, Reino Unido, Croacia) sobre el pensamiento del autor napolitano. Reunión científica mundial que se vería re-alimentada un año después con la aparición de las Actas correspondientes en tres tomos, bella y cuidadosamente editados por la casa italiana La Città del Sole (Nápoles, 2001). Como se puede suponer, las perspectivas de análisis y comparación de las distintas dimensiones o planos del pensamiento de Vico fueron allí exhaustivamente tratadas y relacionadas con los aspectos más modernos, consecuentes, críticos, pluralistas e innovadores del momento, que es decir, en definitiva, de la actualidad. Se nos aparecen así las vertientes más propias del autor, la retórica, la poética, el lenguaje, su concepción sobre el humanismo, la religión, la historia, las ciencias, la política, la filosofía, la antropología, la filosofía de la historia, la historia de las ideas, o la afirmación -como sería nuestra propuesta- de la identidad a través de la inteligencia emocional y la capacidad empática de comprender al otro y ponerse a sí mismo en su lugar. Y todo ello entrecruzándose con distintas tradiciones que asumen un papel importante a desarrollar por nuestro autor por su conocimiento personal en muchos casos o por la incidencia posterior que su pensamiento puede tener y ejercer: así la tradición sefardí, la Escolástica, el Renacimiento humanista, el Barroco, la Ilustración, el siglo XIX y el desarrollo de la ciencia social moderna. El trabajo de preparación de las actas, publicándose los trabajos en diversas lenguas además de en castellano, aunque editadas por empresa napolitana, supuso una visión holística de Vico que nos proporcionaba matices interdisciplinares capaces de traspasar los límites de la objetivación histórica proporcionando interpretaciones absolutamente novedosas, además de originales, que permitían llenar de significado las nuevas direcciones, las nuevas narrativas para el tercer milenio, que intentaban explicar el mundo.

Planteamos además un carácter inherente a la propia filosofía como forma de interpretar científicamente el mundo como era la inteligencia emocional, instrumento empático necesario para llenar de contenido y entender en sus justos términos la filosofía viquiana. 
El cuarto hito en nuestra re-comprensión de Vico pasa por la publicación de la totalidad de las ponencias presentadas en el II Seminario Internacional de Filosofia política: Giambattista Vico y el mundo moderno, celebrado entre los días 22 y 25 de Septiembre de 2003 en la mexicana Universidad Autónoma Metropolitana, cuyos trabajos fueron editados por el hispalense "Centro de Investigaciones sobre Vico" en su órgano Cuadernos sobre Vico, números 17-18 correspondientes a los años 2004-2005, estructurados en seis ámbitos epistemológicos determinados referidos a la Historia, la Filosofía, la Política, la Cultura, la Religión; y uno último correspondiente a la Presencia de Giambattista Vico en Latinoamérica. Congreso de ámbito hispanoamericano, de contenido variado y poliédrico, que debería permitir llegar a interpretar la "visión", la "perspectiva" sobre el Vico político o sobre la filosofía política de nuestro autor, o al menos «comprender su obra desde la filosofía política y la historiografía ${ }^{2}$ que se produce y debate desde América latina y que a pesar del "“revival" de Vico en las últimas décadas ha demostrado que el pensamiento de este excéntrico filósofo italiano está lleno de sorpresas». ${ }^{3}$ Lo más interesante que nos ha aportado esta actividad de la filosofía hispánica en América es la frescura, el rejuvenecimiento, la osadía a veces, de renovados pensadores que a pesar de una formación completa clásica, en muchos aspectos, arriesgan nuevos modos de pensar una tradición, un autor, a través de la aplicación empática del ingenio y de la razón, y desde una tradición propia diferente, abierta y necesitada de otros valores a un pensamiento que aún tiene mucho que revelar en tiempos actuales.

Un último hito, de momento, pues la historia de los Cuadernos sobre Vico sigue abierta, ha sido el número especial para la celebración del XXV aniversario de nuestra revista, número editado por el prof. Sevilla y el autor de esta nota, y en el que con motivo de la celebración se solicitó de nuevo a los más importantes especialistas y expertos en el pensamiento viquiano un trabajo colaborativo en el que se describiese la influencia de la obra del autor napolitano. Estamos convencidos de que este "penúltimo" hito se completará con la aparición del presente número en el que celebramos el $350^{\circ}$ Aniversario del nacimiento del pensador al que dedicamos esta Revista hace ya un cuarto de siglo, y en el que a través de la visión personal, de la influencia íntima, de la seducción intelectual, del descubrimiento de la pasión, del polémico enfrentar día a día lo propio pensado con lo ya propuesto por él, Vico adviene presente, oferta futuro, propone repensar el pasado hasta darle sentido. Visiones, ensoñaciones, interpretaciones de muchos autores a través de los cuales el pensamiento de Vico, aparentemente, se disfraza, se esconde, se oculta,

2. Ramos-Alarcón Marcín, L., "Introducción" en S. Florencia de la Campa, A. Gutiérrez Robles, J. Velázquez Delgado (Coord.), Vico y el Mundo Moderno, UAM, México, 2014, p. 11.

3. GonZÁLEz. C., «Vico y Bacon: La historia de una reconciliación entre los antiguos y los modernos», Cuadernos sobre Vico, n. 17-18, 2004/2005, pp. 89-98, p. 97. 
se solapa, se finge, se disimula, se sobrepone, se cubre, para definitivamente aparecer como un sistema conceptual de validez atemporal y universal.

Además de las mediaciones conceptuales o intelectuales, a través de Vico se nos han dado también ciertas mediaciones personales, que transformadas en entrañables amistades, nos han mostrado de otra forma la filosofía y la filosofía viquiana, a través de los anhelos, las ensoñaciones, las esperanzas de quien quiere comprender y compartir el mundo en toda su cabida temporal y espacial, que quiere interpretar, y al comprender e interpretar nos proporciona los medios para poder hacerlo renovadamente nosotros, en nuestra época. Y estamos recordando ahora a dos amigos y compañeros, precipitadamente fallecidos, y con los cuales y a través de Vico, de su quehacer hecho nuestro, compartimos experiencias vitales a nivel profesional y personal. Nos referimos al profesor Franco Ratto y la profesora Bruna Consarelli, que como en aquel recuerdo juvenil que comentábamos mas arriba, sabiendo ahora bien por qué, siempre relacionaremos con Vico.

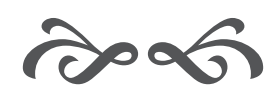


<smiles>[As]</smiles> 